Case Report

\title{
Mesothelioma in Two Nondomestic Felids: North American Cougar (Felis concolor) and Cheetah (Acinonyx jubatus)
}

\author{
Amanda Whiton, ${ }^{1}$ Juergen Schumacher, ${ }^{1}$ Erika E. Evans, ${ }^{1}$ Janelle M. Novak, ${ }^{2}$ \\ Amanda Crews, ${ }^{2,3}$ Edward Ramsay, ${ }^{1}$ and Robert Donnell ${ }^{2}$ \\ ${ }^{1}$ Department of Small Animal Clinical Sciences, College of Veterinary Medicine, University of Tennessee, 2407 River Drive, \\ Knoxville, TN 37996, USA \\ ${ }^{2}$ Department of Biomedical and Diagnostic Sciences, College of Veterinary Medicine, University of Tennessee, \\ Knoxville, TN 37996, USA \\ ${ }^{3}$ Professional Veterinary Pathology Services, Columbia, SC 29203, USA
}

Correspondence should be addressed to Juergen Schumacher; jschumacher@utk.edu

Received 15 April 2013; Accepted 8 May 2013

Academic Editors: L. Espino López, N. D. Giadinis, J. S. Munday, F. Mutinelli, and J. Orós

Copyright (C) 2013 Amanda Whiton et al. This is an open access article distributed under the Creative Commons Attribution License, which permits unrestricted use, distribution, and reproduction in any medium, provided the original work is properly cited.

\begin{abstract}
A 15-year-old male North American cougar (Felis concolor) presented with a 2-day history of anorexia, restlessness, and dyspnea. White blood cell count $\left(22.5 \times 10^{3}\right.$ cells $\left./ \mu \mathrm{L}\right)$ and absolute segmented neutrophil count $\left(21.09 \times 10^{3}\right.$ cells $\left./ \mu \mathrm{L}\right)$ were increased, and BUN $(143 \mathrm{mg} / \mathrm{dL})$, creatinine $(6.3 \mathrm{mg} / \mathrm{dL})$, and phosphorus $(8.5 \mathrm{mg} / \mathrm{dL})$ concentrations indicated chronic renal disease. Thoracic radiographs showed severe pleural and pericardial effusion. During attempts to remove the fluid, cardiac tamponade developed and the cat died. At necropsy, nodular masses decorated the pericardium at the level of the base of the heart. The final microscopic diagnosis was mesothelioma of the pericardium, tunica adventitia of the main pulmonary artery, left auricle epicardium, and left ventricular epicardium. A 15-year-old female cheetah (Acinonyx jubatus) was evaluated for acute respiratory distress. The white blood cell count $\left(25.5 \times 10^{3}\right.$ cells $\left./ \mu \mathrm{L}\right)$ and absolute segmented neutrophil count $\left(22.19 \times 10^{3}\right.$ cells $\left./ \mu \mathrm{L}\right)$ were increased. Radiographically pleural effusion and a cranial thoracic mass were seen. The cheetah was euthanized, and a gross diagnosis of disseminated pleural mesothelioma with thoracic effusion was made. Histologically, pleural mesothelioma was confirmed with local invasion of the lung and pulmonary arterial emboli and infarction. In both cases, a diagnosis of mesothelioma was made based on cellular morphology, microscopic architecture, and neoplastic cell coexpression of cytokeratin and vimentin.
\end{abstract}

\section{Introduction}

Mesothelioma is a neoplasm involving cells that line the coelomic cavities of the body and can occur as either a widespread nodular mass or multifocal masses. Mesothelial tumors are considered malignant due to their ability to seed throughout a body cavity resulting in numerous tumors and implantation metastasis; however, spread into noncommunicating body cavities is considered rare [1]. In domestic dogs, primary mesothelial tumors have been reported affecting the thoracic cavity, pericardial sac, abdominal cavity, and vaginal tunics of the scrotum [1]. In domestic cats, primary mesotheliomas have been reported in the pericardium, pleura, peritoneum, and the abdomen with pulmonary and nodal metastases [1-6]. While pleural mesotheliomas have been reported in tigers and clouded leopards (Neofelis nebulosa nebulosa), pericardial mesotheliomas are rarely reported in both domestic and nondomestic species [7-9].

A common clinical sign of affected animals is pronounced dyspnea caused by pleural effusion or compression from peritoneal effusion. Marked effusions from direct exudation of the tumor or from tumor-obstructed lymphatics can be present. Animals diagnosed with pericardial mesothelioma may present with cardiac tamponade or evidence of rightsided heart failure [1]. A pericardial mesothelioma in a domestic cat with effusion and the clinical presentation and pathologic findings of a pericardial mesothelioma in a Bengal tiger (Panthera tigris) have been reported $[10,11]$. Histologically, mesotheliomas can appear as either epithelial, mesenchymal, or biphasic. 


\section{Case Reports}

Case 1. A 15-year-old male cougar (Felis concolor) from a large felid sanctuary in Tennessee, USA, presented to the University of Tennessee Veterinary Medical Center (UTVMC) with a 2-day history of anorexia, lethargy, and restlessness. Prior to transport to the UTVMC, the cougar received oral midazolam (NovaPlus; Hospira, Lake Forest, IL, USA; $0.12 \mathrm{mg} / \mathrm{kg}$ ). At presentation, the cougar was mildly sedated but alert. The animal was in good body condition (body weight, $62 \mathrm{~kg}$ ). Respiratory rate was 28 breaths per minute, and inspiratory phase was prolonged. Mucous membranes were cyanotic. The animal was immobilized via hand injection with a combination of dexmedetomidine $(0.01 \mathrm{mg} / \mathrm{kg}$ i.m.; DexDomitor; Pfizer Animal Health, Exton, PA, USA) and ketamine hydrochloride (3 mg/kg i.m.; Ketaset; Fort Dodge Animal Health, Fort Dodge, IA, USA). Following endotracheal intubation, anesthesia was maintained with $0.5-2 \%$ isoflurane (Aerrane; Baxter Healthcare Corporation, Deerfield, IL) in $100 \%$ oxygen. Thoracic auscultation revealed bilaterally decreased lung sounds and muffled heart sounds. The femoral pulse was weak, and heart rate was 98 beats per minute. An intravenous catheter was placed in the left medial saphenous vein, and Normosol-R (Hospira) was administered at $10 \mathrm{~mL} / \mathrm{kg} / \mathrm{hr}$. Electrocardiography (ECG) leads were attached in a conventional manner, and no abnormalities were recorded. A transmission pulse oximeter probe was placed over a lingual artery, and oxygen saturation $\left(\mathrm{SpO}_{2}\right)$ values were $<90 \%$, indicating severe hypoxemia. End-tidal carbon dioxide $\left(\mathrm{EtCO}_{2}\right)$ concentrations were $>40 \mathrm{mmHg}$, indicative of hypercapnia. In order to antagonize the effects of dexmedetomidine and improve cardiopulmonary performance, intramuscular atipamezole (Antisedan; Pfizer Animal Health, New York, NY, USA; $0.05 \mathrm{mg} / \mathrm{kg}$ ) was administered 10 minutes after endotracheal intubation. A venous blood sample was collected from the jugular vein and submitted for hematology, plasma biochemistry, and feline coronavirus (FCoV) titers.

Right lateral and dorsoventral radiographs of the thorax revealed rounding of the lung lobes and separation of the lung lobes from the body wall by a soft tissue opacity, consistent with pleural effusion. Air bronchograms were seen in the right cranial and right middle lung lobes. Cardiac margins were not seen, and there was a mild right mediastinal shift. Abdominal radiographs showed decreased serosal detail and mottling falciform and omental fat. An approximately $2 \mathrm{~cm}$ diameter soft tissue mineral opaque nodule with irregular margins was present over the plane of the liver on lateral radiographs. The radiographic interpretation was bicavitary effusion secondary to a neoplastic process, cardiac disease, or infectious process.

Thoracocentesis revealed a yellow, cloudy, and highly cellular fluid with a total protein of $3.4 \mathrm{~g} / \mathrm{dL}$. Small lymphocytes were the predominant cells, but there were also low numbers of foamy macrophages that occasionally exhibited erythrophagocytosis and contained small amounts of hemosiderin-like material. A cytologic diagnosis of a lymphocyte-rich, probable chylous effusion with the presence of red blood cells indicative of prior hemorrhage and increased

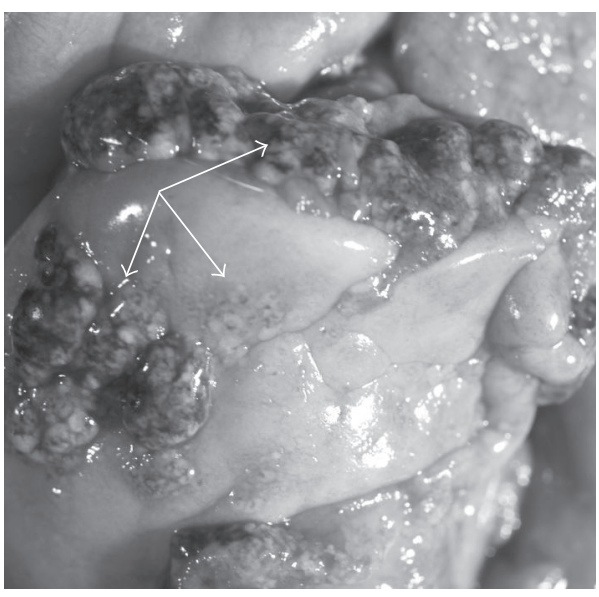

FIGURE 1: Gross photograph of numerous neoplastic mesothelial, exophytic, and tan to brown masses (arrows) on the epicardial and visceral pericardial surfaces of a 15 -year-old cougar (Felis concolor).

numbers of mast cells was made. During removal of approximately $2 \mathrm{~L}$ fluid from the chest, the cougar developed ventricular premature complexes, became tachycardic, and arrested. Resuscitation efforts were unsuccessful.

Blood work abnormalities included an elevated white blood cell count $\left(22.5 \times 10^{3}\right.$ cells $/ \mu \mathrm{L}$; reference intervals, $3.10-16.90 \times 10^{3}$ cells $\left./ \mu \mathrm{L}\right)$ and an elevated absolute segmented neutrophil count $\left(21.09 \times 10^{3}\right.$ cells $/ \mu \mathrm{L}$; reference interval, $0.056-11.60 \times 10^{3}$ cells $\left./ \mu \mathrm{L}\right)$. BUN $(143 \mathrm{mg} / \mathrm{dL}$; reference interval, $12-65 \mathrm{mg} / \mathrm{dL})$, creatinine $(6.3 \mathrm{mg} / \mathrm{dL}$; reference interval, $0.8-5.3 \mathrm{mg} / \mathrm{dL})$, and phosphorus $(8.5 \mathrm{mg} / \mathrm{dL})$ levels were also markedly increased, indicating chronic renal disease. Serum was negative for antibodies to FCoV. Aerobic and anaerobic cultures of the thoracic fluid yielded no bacterial growth. Samples from the thoracic and abdominal effusion were PCR negative for FCoV.

At necropsy, the thoracic cavity contained $3 \mathrm{~L}$ of mildly gelatinous serosanguineous fluid. There was diffuse pulmonary atelectasis. Multifocal to coalescing petechial luminal hemorrhages were located throughout the length of the trachea. Fibrinous adhesions were present between the parietal pleura, pericardial/mediastinal pleura, and pulmonary pleura. The wall of the pericardial sac was opaque, thickened, and edematous. The pericardial sac contained $600 \mathrm{~mL}$ thick red to purple fluid. Numerous, brown exophytic proliferations, ranging in size from $0.5 \times 0.2 \times 0.3$ to $5.0 \times 2.0 \times 1.5 \mathrm{~cm}$, covered the epicardial surface of both atria, encircled the main pulmonary artery and aorta, extended to the cardiac apex, and were continuous with masses present on the visceral surface of the pericardium (Figure 1). A $1.0 \times 1.0 \times$ $0.5 \mathrm{~cm}$ gray to white focal mass expanded the mediastinum. One liter of red-orange-yellow gelatinous fluid was found within the abdominal cavity. The spleen was enlarged, and the gallbladder was distended with a torturous cystic duct.

Histopathologic evaluation of the main pulmonary artery (with exophytic proliferations) revealed an unencapsulated population of polygonal to columnar cells arranged in undulating folds, papillary projections, and dense sheets on 
a fibrovascular stroma. Along the main pulmonary artery, the neoplastic cells proliferated along the tunica adventitia and blended with adipose tissue (Figure 3(a)). These neoplastic cells incorporated the epicardium and blended with the surrounding epicardial adipose tissue. There was histopathologic evidence of pleural and visceral pericardial involvement. Neoplastic cells had oval euchromatic nuclei, eosinophilic cytoplasm, and indistinct borders. There was moderate anisocytosis (2-3x) and anisokaryosis (2-3x). Mitotic figures averaged 1/10 high-power fields (400x). There was multifocal necrosis with amorphous eosinophilic material and karyorrhectic debris with scattered dystrophic mineralization. Mixed with neoplastic cells were neutrophils, lymphocytes, plasma cells, epitheloid macrophages, siderophages, and hemorrhage. Immunohistochemical staining revealed the presence of both cytokeratin (clones AE1/AE3, DAKO) and vimentin (clone V9, DAKO) intermediate filaments in the neoplastic cells (Figures 3(b) and 3(c)). Other relevant histopathologic findings in the lungs included alveolar histiocytosis with "heart failure cells," alveolar smooth muscle hypertrophy, and hyperplasia with type II pneumocyte hypertrophy, all related to cardiac insufficiency.

The diagnosis was chronic active pericarditis and mesothelioma involving the pericardium, tunica adventitia of the main pulmonary artery, left auricle epicardium, and left ventricular epicardium.

Case 2. A 15-year-old female cheetah (Acinonyx jubatus) (body weight, $41.5 \mathrm{~kg}$ ) from a zoological institution in Tennessee, USA, presented with acute onset of abdominal breathing and a 1-day history of lethargy and anorexia. The cheetah was immobilized with an intramuscular combination of ketamine $(3.5 \mathrm{mg} / \mathrm{kg}$; Ketaset; Fort Dodge Animal Health), dexmedetomidine $(0.012 \mathrm{mg} / \mathrm{kg}$, DexDomitor; Pfizer Animal Health), and midazolam (0.06 mg/kg, NovaPlus, Hospira) via a remote drug delivery system. Following endotracheal intubation, anesthesia was maintained with $2 \%$ isoflurane in $100 \%$ oxygen. An intravenous catheter was placed in the cephalic vein, and Normosol-R was administered at $10 \mathrm{~mL} / \mathrm{kg} / \mathrm{hr}$. A venous blood sample was collected from the jugular vein and submitted for hematology, plasma biochemistry, and FCoV serology.

Thoracic radiographs revealed a cranial thoracic mass and separation of the lung lobes from the body wall, consistent with pleural effusion. Differential diagnoses included lymphoma, thymoma, lymphadenopathy, and thyroid carcinoma. Fluid was also noted in the abdominal cavity. The animal was euthanized due to quality of life concerns and poor prognosis for recovery.

Cytology of the thoracic effusion detected round cells and unidentifiable clusters, which most likely represented mesotheliocytes; however, some features of malignancy, for example, carcinoma, could not be ruled out. Aerobic and anaerobic cultures of the thoracic nodules revealed one colony of a gram-negative, nonfermentative rod, resembling Acinetobacter sp. Bacterial culture of a venous blood sample revealed no organisms on a gram stain, no anaerobes, and few colonies of Klebsiella pneumoniae and actinomyces-like organisms.

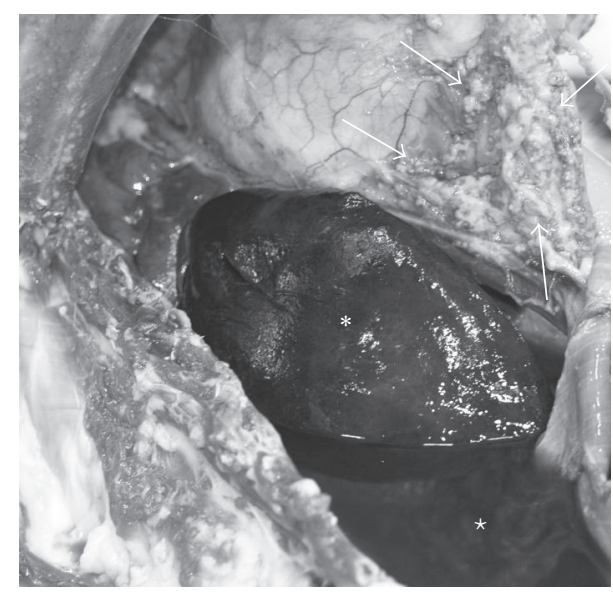

FIGURE 2: Gross photograph of the right thoracic cavity of a 15-yearold cheetah (Acinonyx jubatus). There is engorgement of the torsed right middle lung lobe (asterisk), and serosanguineous fluid partially fills the chest cavity (star). Multifocal coalescing tan nodules (mesothelioma) are present on the pleural and mediastinal surface (arrows).

Hematologic abnormalities included an elevated white blood cell count $\left(25.5 \times 10^{3}\right.$ cells $/ \mu \mathrm{L}$; reference range, $3.70-25.20 \times 10^{3}$ cells $/ \mu \mathrm{L}$ ) and an elevated absolute segmented neutrophil count $\left(22.19 \times 10^{3}\right.$ cells $/ \mu \mathrm{L}$; reference range, $1.34-20.90 \times 10^{3}$ cells $/ \mu \mathrm{L}$ ). Plasma chemistry and electrolytes values were within normal limits, and serum was negative for antibodies to FCoV.

At necropsy, $2 \mathrm{~L}$ of dark red, serosanguineous, and turbid fluid was present within the thoracic cavity (Figure 2). Throughout the chest cavity, small $(0.1-0.5 \mathrm{~cm})$, and multifocal, coalescing white to light tan nodules were firmly attached to the pleura. The pericardial sac contained $4.5 \mathrm{~mL}$ of clear, light yellow fluid. The right middle lobe was turgid, enlarged, dark red, firm, and torsed 360 degrees. The remaining lung lobes were atelectatic. The left cranial lung lobe, toward the apex, contained multifocal irregular areas that were depressed, firm, and dark red with measurements ranging from $0.5 \times 0.2 \times 0.2 \mathrm{~cm}$ to $3.0 \times 1.0 \times 1.8 \mathrm{~cm}$. The right adrenal gland had decreased corticomedullary distinction, was irregular and diffusely enlarged, and measured $3.9 \times$ $2.5 \times 1.3 \mathrm{~cm}$. The adrenal cortex was enlarged, with a corticomedullary ratio of $1.5: 1: 1.4$. The left adrenal gland measured $3 \times 1.5 \times 1 \mathrm{~cm}$.

The gross diagnosis was disseminated pleural mesothelioma with severe thoracic effusion, diffuse pulmonary atelectasis, and acute right middle lung lobe torsion. Additional diagnoses included hepatic congestion, mild multifocal hepatic telangiectasia, multifocal pancreatic ductular cysts, multifocal pancreatic exocrine nodular hyperplasia, adrenal cortical adenoma, multifocal splenic myelolipomas, moderate subacute multifocal gastric congestion, and focal ovarian bursal cyst.

Microscopically, the thoracic mass was lined by raised to exophytic aggregates of moderately pleomorphic mesothelial cells. The mass was composed of clusters, cords, and islands of 


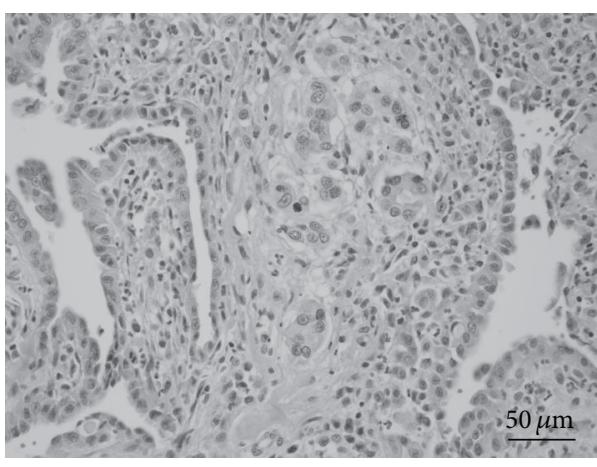

(a)

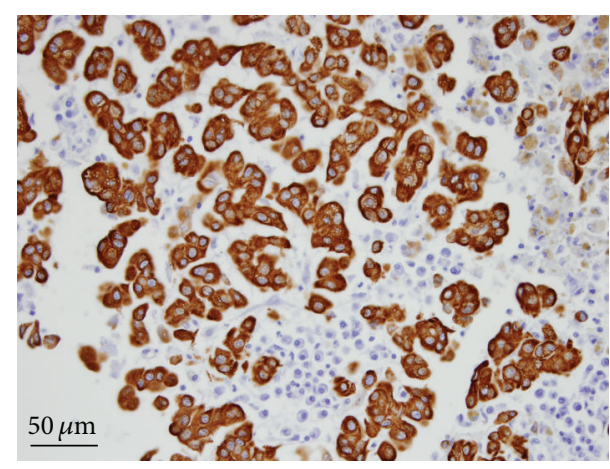

(b)

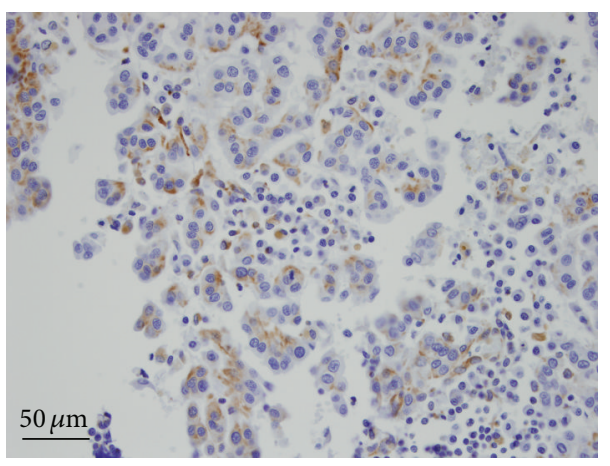

(c)

FIGURE 3: Photomicrographs of a mass overlying the epicardium and main pulmonary artery from a 15-year-old cougar. (a) Multiple papillary structures of fibrovascular stroma and nests of anisocytotic pleomorphic polygonal cells are covered by pleomorphic anisocytotic cuboidal to columnar cells (hematoxylin and eosin stain). (b) Clustered and individual cells strongly stain brown for the presence of cytokeratin intermediate filaments (immunohistochemistry, cytokeratin [AE1/AE3] antibody, DAB chromagen, hematoxylin counterstain). (c) The same clustered and individual cells stain brown for the presence of vimentin intermediate filaments (immunohistochemistry, vimentin [V9] antibody, DAB chromagen, hematoxylin counterstain). All figures are at 40x objective. Bar $=50 \mu \mathrm{M}$.

polygonal cells confluent with the surface cells. The neoplastic cells had variably distinct cell borders, moderate amphophilic cytoplasm, round euchromatic nuclei with finely stippled chromatin, and prominent nucleoli. Anisocytosis was moderate (2-3x), and anisokaryosis was marked (4-5x). Mitotic figures averaged 3/10 high-power fields (400x). Scattered throughout the tissue were areas of necrosis with neutrophils, lymphocytes, hemorrhage, and amphophilic-fibrillar material. Neoplastic cells exhibited staining for antibody binding to both cytokeratin and vimentin intermediate filaments.

The pulmonary pleura had multiple focal to extensive, superficial to infiltrative aggregates of moderately pleomorphic polygonal cells. Within the left cranial lung lobe, two vessels (arteries, presumptive) contained the same neoplastic cells as previously noted, proximal to a large area of hemorrhage admixed with cellular debris and fibrin extending to the apex (infarction) with diffuse pleural fibrosis.

Sections of the diaphragm had serosal proliferation and diaphragmatic infiltration by the neoplastic cells described above. Subjacent myocytes were shrunken and hypereosinophilic.

The right adrenal gland was effaced by a large area of necrosis surrounded by highly infiltrative cells as described for the pleural lesion. These extended through the cortical parenchyma with a reactive fibrous connective tissue proliferation on the outer surface. The zona reticularis was thickened in the left adrenal gland. The histopathologic diagnosis was disseminated pleural mesothelioma with local invasion of the lung; pulmonary arterial emboli; and adrenal, diaphragmatic, and hepatic metastases.

A final diagnosis of mesothelioma with marked thoracic effusion and adrenal metastasis was made based on cellular morphology and coexpression of cytokeratin and vimentin.

\section{Discussion}

In both the cougar and cheetah, a diagnosis of mesothelioma was made based on cytologic, histologic, and immunohistochemical findings. The cougar was diagnosed with pericardial mesothelioma, which is considered rare in all species, and to the authors' knowledge, this is only the second report of a primary pericardial mesothelioma in a large cat [12]. A diagnosis of disseminated pleural mesothelioma was made in the cheetah. Pleural mesotheliomas in large cats have been reported in both tigers and clouded leopards $[8,12]$.

While mesothelioma in humans and rats is often associated with asbestos exposure [1], this has yet to be definitively associated with the formation of mesothelioma in domestic and nondomestic animals. A study in dogs found increased 
levels of asbestos in lung tissues of affected animals versus control dogs; however, this has not been documented in domestic cats [13]. In animals, exposure to asbestos may be only a contributing factor for the development of mesothelioma. Golden retrievers developed pericardial mesothelioma after a long history of idiopathic hemorrhagic pericardial effusion, suggesting that chronic inflammation may also lead to neoplastic transformation [14]. Mesothelial tumors are most often associated with older animals, but reports of dogs as young as 7 weeks of age suggest that a congenital form may also be present [1]. While there is a report of pleural mesothelioma in a female lion housed within an enclosure in which asbestos fibers were found, no fibers were identified in either the lung or neoplastic tissues [7]. Although not definitive, that report suggests that asbestos exposure may lead to mesothelioma formation in animals as well. Additionally, asbestos fibers are small and can be easily cleared, making them difficult to detect within sections of the lung. There was no known exposure to asbestos in either the cougar or cheetah in the present cases.

Both the cheetah and cougar were diagnosed with tricavitary effusion. Possible causes of tri-cavitary effusion include congestive heart failure, hypoproteinemia (renal and liver disease, glomerulonephritis, malabsorption, and parasitism), neoplasia, bacterial peritonitis, feline infectious peritonitis (FIP), pansteatitis, toxoplasmosis, tuberculosis, pregnancy, and trauma. An important diagnostic ruleout in feline species is FIP caused by mutation of FCoV, which affects both wild and domestic cats. Serum that is positive for FCoV is highly suggestive, but not a definitive diagnosis, of FIP [15]. Cheetahs in particular are known to be susceptible to FCoV. Both felids in this report were negative for FCoV.

Intrathoracic masses, such as mesotheliomas, can cause chylothorax due to thoracic duct obstruction, which prevents lymph draining into the vena cava. Chylothorax is an accumulation of lymph in the pleural space; it appears milky and has a high triglyceride and lymphocyte content. This accumulation of fluid in the thorax can in turn cause pulmonary atelectasis, as seen in the cougar in this report. Histologically, within the lung of the cougar were increased numbers of macrophages containing hemosiderin consistent with "heart failure cells," likely a result of restriction due to pericardial fluid accumulation.

The diagnosis of mesothelioma in the cheetah was based on the extensive involvement of the pleura with no evidence of a primary carcinoma elsewhere. While the adrenal involvement raised the possibility of an adrenal carcinoma metastasizing to the pleural cavity with carcinomatosis, at the time of gross examination, the histologic findings supported a diagnosis of mesothelioma rather than carcinoma.

A clinical diagnosis of mesothelioma can be difficult and challenging, as it is mainly a diagnosis of exclusion. Clinical presentation of the cougar and cheetah reported here differed when comparing respiratory status. The cheetah presented in respiratory distress, while the cougar's clinical signs were mild and associated with an increased inspiratory phase only. Both animals had radiographic evidence of bicavitary effusion, which was confirmed at necropsy.
Based on the findings of the cases reported here, mesothelioma should be suspected in patients presenting with chronic, nonspecific disease and fluid accumulation in either the thoracic and/or abdominal cavity. Radiography and ultrasonography are not considered the diagnostic imaging modalities of choice due to the presence of effusion, which will make detection of masses difficult. Thoracic/abdominal computed tomography is more sensitive for identifying nodular lesions in the presence of effusion.

Cytologic evaluation of fluid is of diagnostic value for identification of thoracic disease processes. Although mesothelial cells are commonly identified in fluid, these cells will proliferate in multiple pathologic conditions associated with fluid accumulation in a body cavity. This feature makes it difficult to determine whether the mesothelial cell proliferation is reactive or neoplastic. Cytologic evaluation of thoracic effusion obtained via thoracocentesis in the cougar did not demonstrate mesothelial cells but a lymphocyte-rich effusion and increased numbers of mast cells. In the cheetah, round cells and clusters of cells most likely representing reactive mesotheliocytes with some features of malignancy were identified upon cytologic evaluation of the thoracic fluid. Pericardiocentesis in a Bengal tiger with pericardial mesothelioma yielded an extremely cellular hemorrhagic effusion (specific gravity of 1.033), containing reactive mesothelial cells and erythrocytes [11].

Gross evidence of disease differed between the two cases as well. On necropsy, the cougar's final diagnosis was pericardial mesothelioma, whereas pleural mesothelioma with adrenal metastasis was evident in the cheetah. For the cheetah, adrenal carcinoma metastasizing to the pleural cavity with associated carcinomatosis could not be completely ruled out; however, this is considered unlikely.

Histologically, it is important to differentiate mesothelioma from carcinomas, adenocarcinomas, or sarcomas, based on the morphologic type of mesothelioma. Immunohistochemistry to determine biphasic intermediate filament expression is diagnostically useful in that cytokeratin expression is often positive in mesotheliomas and therefore is helpful in differentiating sarcomatous mesotheliomas from sarcomas [1]. Vimentin expression is of diagnostic value in differentiating epithelial mesothelioma from adenocarcinomas, which are typically vimentin-negative [7]. In both animals of this report, sections of the masses were positive for both cytokeratin and vimentin, which further supports a diagnosis of mesothelioma. Although not performed in these cases, the use of calretinin antibody may be beneficial in differentiating between mesotheliomas and adenocarcinomas. Immunohistochemistry demonstrating the concurrent presence of cytokeratin and vimentin played an important role in the final diagnosis of mesothelioma in both cases. A diagnosis of mesothelioma is based on a tumor associated with the lining of the coelomic cavity, presence/absence of effusion, histopathologic differentiation of cell types, and immunohistochemical staining [1]. In most cases, the etiology of the tumor is unidentified, with affected animals having no known exposure to potential causative agents. 


\section{Acknowledgments}

The authors thank the carnivore staff at the Knoxville Zoological Gardens and Tiger Haven, Kingston, TN, USA, in particular M. L. Haven and D. Chaffins for their care and assistance with the animals presented in this report.

\section{References}

[1] L. Garrett, "Mesothelioma," in Small Animal Clinical Oncology, S. Withrow and D. Vail, Eds., pp. 804-808, Elsevier, St. Louis, Mo, USA, 2007.

[2] C. P. Raflo and S. P. Nuernberger, "Abdominal mesothelioma in a cat," Veterinary Pathology, vol. 15, no. 6, pp. 781-783, 1978.

[3] A. N. Al-Dissi and H. Philibert, "A case of biphasic mesothelioma with osseous and chondromatous differentiation in a cat," Canadian Veterinary Journal, vol. 52, no. 5, pp. 534-536, 2011.

[4] B. Bacci, F. Morandi, M. de Meo, and P. S. Marcato, "Ten cases of feline mesothelioma: an immunohistochemical and ultrastructural study," Journal of Comparative Pathology, vol. 134, no. 4, pp. 347-354, 2006.

[5] T. M. J. Heerkens, J. D. Smith, L. Fox, and J. M. Hostetter, "Peritoneal fibrosarcomatous mesothelioma in a cat," Journal of Veterinary Diagnostic Investigation, vol. 23, no. 3, pp. 593-597, 2011.

[6] Y. Kobayashi, H. Usuda, K. Ochiai, and C. Itakura, "Malignant mesothelioma with metastases and mast cell leukaemia in a cat," Journal of Comparative Pathology, vol. 111, no. 4, pp. 453-458, 1994.

[7] E. Bollo, F. E. Scaglione, M. Tursi et al., "Malignant pleural mesothelioma in a female Lion (Panthera leo)," Research in Veterinary Science, vol. 91, no. 1, pp. 116-118, 2011.

[8] A. A. Cunningham and A. P. Dhillon, "Pleural malignant mesothelioma in a captive clouded leopard: (Neofelis nebulosa nebulosa)," Veterinary Record, vol. 143, no. 1, pp. 22-24, 1998.

[9] N. S. Shin, S. W. Kwon, D. Y. Kim, O. K. Kweon, I. B. Seo, and J. H. Kim, "Metastatic malignant mesothelioma in a tiger (Panthera tigris)," Journal of Zoo and Wildlife Medicine, vol. 29, no. 1, pp. 81-83, 1998.

[10] L. P. Tilley, J. M. Owens, R. J. Wilkins, and A. K. Patnaik, "Pericardial mesothelioma with effusion in a cat," Journal of the American Animal Hospital Association, vol. 11, no. 1, pp. 60-65, 1975.

[11] E. B. Wiedner, R. Isaza, W. A. Lindsay, A. L. Case, J. Decker, and J. Roberts, "Pericardial mesothelioma in a Bengal tiger (Panthera tigris)," Journal of Zoo and Wildlife Medicine, vol. 39, no. 1, pp. 121-123, 2008.

[12] A. Th. Weiss, A. B. da Costa, and R. Klopfleisch, "Predominantly fibrous malignant mesothelioma in a cat," Veterinary Medicine International, vol. 2010, Article ID 396794, 4 pages, 2010.

[13] L. T. Glickman, L. M. Domanski, T. G. Maguire, R. R. Dubielzig, and A. Churg, "Mesothelioma in pet dogs associated with exposure of their owners to asbestos," Environmental Research, vol. 32, no. 2, pp. 305-313, 1983.

[14] N. Machida, R. Tanaka, N. Takemura, Y. Fujii, A. Ueno, and K. Mitsumori, "Development of pericardial mesothelioma in golden retrievers with a long-term history of idiopathic haemorrhagic pericardial effusion," Journal of Comparative Pathology, vol. 131, no. 2-3, pp. 166-175, 2004.

[15] L. W. Myrrha, F. M. F. Silva, E. F. Peternelli, A. S. Junior, M. Resende, and M. R. Almeida, "The paradox of feline coronavirus pathogenesis: a review," Advances in Virology, vol. 2011, Article ID 109849, 8 pages, 2011. 

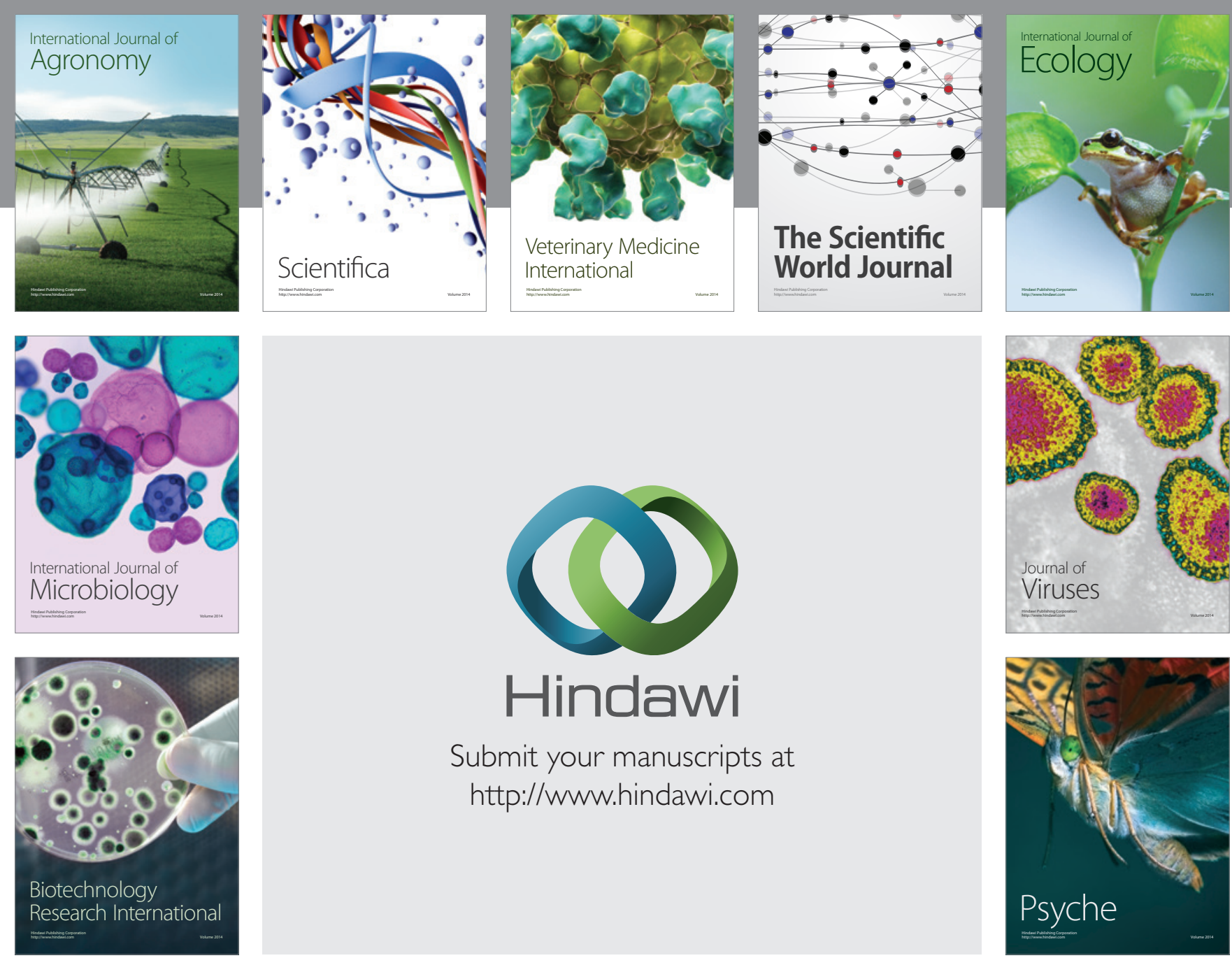

Submit your manuscripts at http://www.hindawi.com
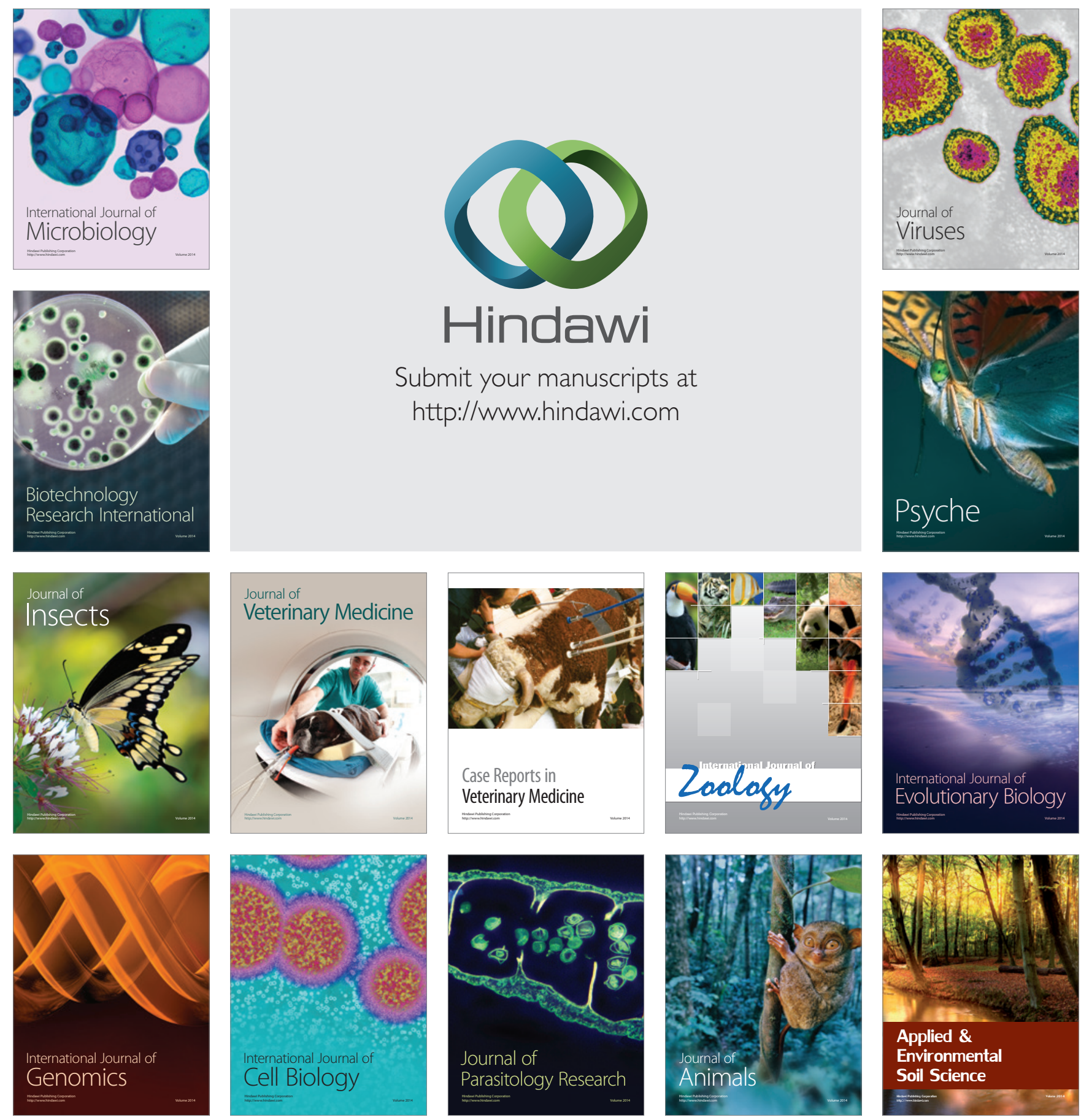